\title{
Quantifying Pancreatic Islet Architecture with Confocal Fluorescence Microscopy: Endocrine Cell Type Distribution Effects on Hormone Secretion
}

Courtney M. Richman ${ }^{1}$ and David W. Piston ${ }^{1}$

${ }^{1}$ Department of Cell Biology and Physiology, Washington University in St. Louis, St. Louis, MO, USA

Coordination among insulin-secreting $\beta$ cells, glucagon-secreting $\alpha$ cells, and somatostatin-secreting $\delta$ cells within the pancreatic islets of Langerhans is required for the maintenance of blood glucose homeostasis. Disrupted secretion patterns result in serious pathophysiology, most notably diabetes. As incidence of diabetes rapidly rises, it is increasingly imperative to investigate the mechanisms involved in glucose regulation. Due to the deleterious effects of hyperglucagonemia in diabetes, particular focus on the regulation of $\alpha$ islet cell secretion is merited. Paracrine-based regulation has been suggested by the observation of somatostatin and insulin-mediated inhibition of glucagon secretion [1]. Through the assessment of the spatial relationships between islet endocrine cells, this study produces data that support a model of proximity-mediated intercellular modulation of islet hormone secretion.

This analysis was achieved through confocal fluorescence microscopy of mouse and human islets. Mouse islets were isolated from B6 mice and human islets were obtained from the NIH-funded Integrated Islet Distribution Program. The islets were fixed in 4\% paraformaldehyde with $0.5 \%$ Triton-X 100. Islets were incubated with primary antibodies targeting insulin, glucagon, and somatostatin and subsequently with Alexa Flour secondary antibodies. Z-stack images of the islets were produced using multichannel confocal imaging on a LSM Zeiss 880. These images were analyzed with ImageJ software by identifying individual cells through stereological cell counting, labeling these cells based on the fluorescent hormone stain, and considering both 3D coordinates and image stacks to assess proximity between $\alpha$ cells, $\delta$ cells, and $\alpha$ and $\delta$ cells.

The analysis of both islet populations (mouse: $n=5,8$ islets; human $n=2,4$ islets) supported previously reported data about islet composition. Qualitatively, mouse and human islets demonstrated architectural distinctions in their cell type distribution, with $\alpha$ and $\delta$ cells concentrated in the mantle of mouse islets and more widely dispersed in human islets (Figure 1). Quantitatively, the relative proportions of each cell type were within the expected range for each species [2]. These data helped to verify the accuracy of the analysis methods and provided useful information for the interpretation of cell type distribution data.

Specific focus was directed at quantifying the cellular microenvironment of $\alpha$ and $\delta$ islet cells.

Significantly, the trends in islet cell type distribution were largely consistent between mouse and human islets (Figure 2). These data support the hypothesis that immediate proximity of endocrine cell types is essential to the function and normal secretion of islets across species. Furthermore, these results have interesting implications for understanding the regulation of glucagon secretion from $\alpha$ cells.

In regard to possible regulatory mechanisms, the high specificity for $\alpha-\alpha$ associations is significant. In mouse islets, the average percentage of $\alpha$ cells bordered by at least one other $\alpha$ cell was $91.1 \%$. In human islets this number was $94.9 \%$. While electrical coordination of glucagon secretion has not been observed in $\alpha$ cells as it has for insulin secretion from $\beta$ cells [3], clustering of $\alpha$ cells in islets suggests that their association is important to their function, whether this effect be mediated through electrical coupling or some other mode of coordination. 
The observed association between $\alpha$ and $\delta$ cells is also noteworthy. The percentages of $\delta$ cells bordered by $\alpha$ cells ( $81 \%$ in mouse, $70 \%$ in human) and $\alpha$ cells bordered by $\delta$ cells $(68.5 \%$ in mouse, $62.5 \%$ in human) suggest a reciprocal specificity for $\alpha$ and $\delta$ cell associations. These associations indicate the likelihood of an immediate paracrine effect of locally-secreted somatostatin on $\alpha$ cell activity and introduce the possibility of modulatory juxtacrine interactions between these cell types.

These findings are particularly interesting in conjunction with the known inhibitory effect of somatostatin on glucagon secretion and the reported activity patterns of $\alpha$ cell populations. It has been observed that only about $35 \%$ of $\alpha$ cells are active in mouse islets [4]. This number is correlated with the reported frequency of $\alpha$ cells bordered by $\delta$ cells in the species $(68.5 \%)$, suggesting that spatial association with $\delta$ cells may keep $\alpha$ cells inactive. No comparable data exists for human islets, for which this hypothesis would predict a higher level of active $\alpha$ cells based on a slightly decreased $\alpha-\delta$ cell association. Continued research on the effect of islet architecture on secretion activity will enable testing of this hypothesis and contribute to the elucidation of the mechanisms regulating glucagon secretion.

References:

[1] AD Elliott, A Ustione, and DW Piston. Am J Physiol Endocrinol Metab, 308 (2015), p. 130-43.

[2] M Brissova et al, Journal of Histochemistry \& Cytochemistry, 53 (2005), p. 1087-1097.

[3] RKP Benninger and DW Piston, Trends in Endocrinology \& Metabolism, 25 (2014), p. 399-406.

[4] SJ Le Marchand and DW Piston, Journal of Biological Chemistry, 285 (2010), p.14389-14398.

[5] The authors acknowledge funding from the Microscopy Society of America through the Undergraduate Research Scholarship Program.
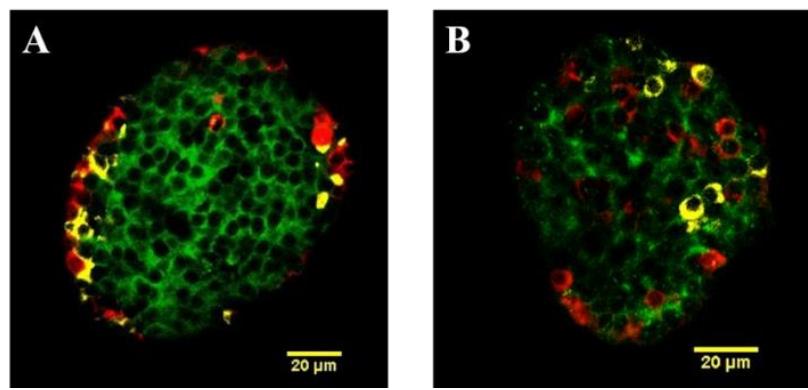

Figure 1. Immunofluorescence of pancreatic islet sections from mouse (A) and human (B) labeled for insulin (green), glucagon (red), and somatostatin (yellow).

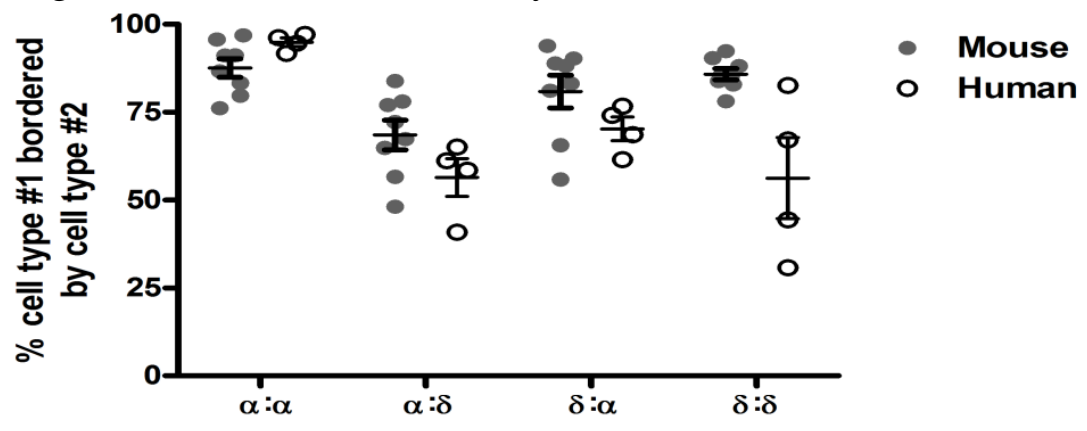

Figure 2. Relationship between islet cell types in mouse and human, where $\alpha: \delta$ reports the $\%$ of $\alpha$ cells bordered by at least one $\delta$ cell. 